\title{
Model independent constraints on transition redshift
}

To cite this article: J.F. Jesus et al JCAP05(2018)073

View the article online for updates and enhancements.

\section{Related content}

Bayesian analysis of CCDM models J.F. Jesus, R. Valentim and F. AndradeOliveira

(t) cosmology induced by a slowly varying Elko field S.H. Pereira, A. Pinho S.S., J.M. Hoff da Silva et al.

Reconstructing the cosmic expansion history with a monotonicity prior Youhua Xu, Hu Zhan and Yeuk-Kwan Edna Cheung

\section{Recent citations}

- Quantifying the evidence for the current
$\frac{\text { speed-up of the Universe with low and }}{\text { intermediate-redshift data. A more model- }}$
independent approach
Adrià Gómez-Valent
- Observational Constraints on the Tilted
Spatially Flat and the Untilted Nonflat CDM
Dynamical Dark Energy Inflation Models
Chan-Gyung Park and Bharat Ratra

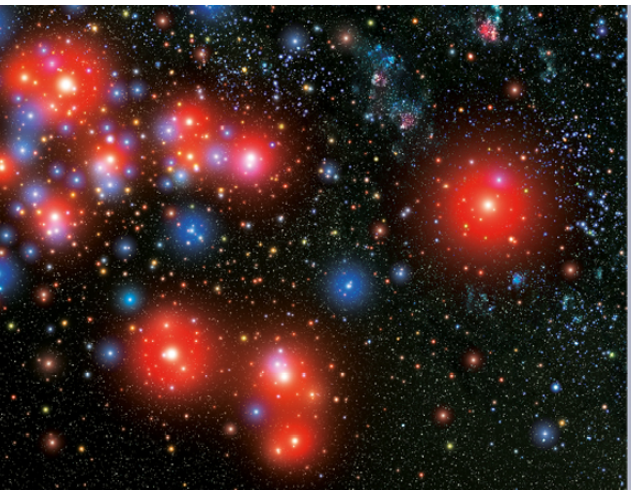

\section{A.A IOP Astronomy ebooks}

Part of your publishing universe and your first choice for astronomy, astrophysics, solar physics and planetary science ebooks. iopscience.org/books/aas 


\title{
Model independent constraints on transition redshift
}

\author{
J.F. Jesus, ${ }^{a, b}$ R.F.L. Holanda ${ }^{c, d, e}$ and S.H. Pereira ${ }^{b}$ \\ ${ }^{a}$ Universidade Estadual Paulista (Unesp), Campus Experimental de Itapeva, \\ R. Geraldo Alckmin 519, Itapeva, SP, 18409-010 Brazil \\ ${ }^{b}$ Universidade Estadual Paulista (Unesp), Faculdade de Engenharia de Guaratinguetá, \\ Departamento de Física e Química, Av. Dr. Ariberto Pereira da Cunha 333, \\ Guaratinguetá, SP, 12516-410 Brazil \\ ${ }^{c}$ Departamento de Física Teórica e Experimental, \\ Universidade Federal do Rio Grande do Norte, \\ Av. Sen. Salgado Filho 3000, Natal, RN, 59078-970 Brazil \\ ${ }^{d}$ Departamento de Física, Universidade Federal de Sergipe, \\ Av. Marechal Rondon S/N, São Cristovão, SE, 49100-000 Brazil \\ ${ }^{e}$ Departamento de Física, Universidade Federal de Campina Grande, \\ R. Aprígio Veloso 882, Campina Grande, PB, 58429-900 Brazil \\ E-mail: jfjesus@itapeva.unesp.br, holanda@uepb.edu.br, shpereira@feg.unesp.br
}

Received December 5, 2017

Revised March 26, 2018

Accepted May 17, 2018

Published May 30, 2018

\begin{abstract}
This paper aims to put constraints on the transition redshift $z_{\mathrm{t}}$, which determines the onset of cosmic acceleration, in cosmological-model independent frameworks. In order to perform our analyses, we consider a flat universe and assume a parametrization for the comoving distance $D_{\mathrm{C}}(z)$ up to third degree on $z$, a second degree parametrization for the Hubble parameter $H(z)$ and a linear parametrization for the deceleration parameter $q(z)$. For each case, we show that type Ia supernovae and $H(z)$ data complement each other on the parameter space and tighter constrains for the transition redshift are obtained. By combining the type Ia supernovae observations and Hubble parameter measurements it is possible to constrain the values of $z_{\mathrm{t}}$, for each approach, as $0.806 \pm 0.094,0.870 \pm 0.063$ and $0.973 \pm 0.058$ at $1 \sigma$ c.l., respectively. Then, such approaches provide cosmological-model independent estimates for this parameter.
\end{abstract}

Keywords: redshift surveys, supernova type Ia - standard candles

ArXiv ePrint: 1712.01075 


\section{Contents}

1 Introduction $\quad 1$

2 Basic equations $\quad 3$

$2.1 z_{\mathrm{t}}$ from comoving distance, $D_{\mathrm{C}}(z) \quad 4$

$2.2 z_{\mathrm{t}}$ from $H(z) \quad 4$

$\begin{array}{lll}2.3 & z_{\mathrm{t}} \text { from } q(z) & 6\end{array}$

3 Samples

$3.1 H(z)$ data

$\begin{array}{lll}3.2 & \text { JLA SNe Ia compilation } & 7\end{array}$

4 Analyses and results $r$

5 Conclusion

\section{Introduction}

The idea of a late time accelerating universe is indicated by type Ia supernovae (SNe Ia) observations [1-8] and confirmed by other independent observations such as Cosmic Microwave Background (CMB) radiation [9-11], Baryonic Acoustic Oscillations (BAO) [12-16] and Hubble parameter, $H(z)$, measurements [17-19]. The simplest theoretical model supporting such accelerating phase is based on a cosmological constant $\Lambda$ term $[20,21]$ plus a Cold Dark Matter component [22-24], the so-called $\Lambda$ CDM model. The cosmological parameters of such model have been constrained more and more accurately $[11,18,25]$ as new observations are added. Beyond a constant $\Lambda$ based model, several other models have been also suggested recently in order to explain the accelerated expansion. The most popular ones are based on a dark energy fluid $[26,27]$ endowed with a negative pressure filling the whole universe. The nature of such exotic fluid is unknown, sometimes attributed to a single scalar field or even to mass dimension one fermionic fields. There are also modified gravity theories that correctly describe an accelerated expansion of the Universe, such as: massive gravity theories [28], modifications of Newtonian theory (MOND) [29-31], $f(R)$ and $f(T)$ theories that generalize the general relativity [32-34], models based on extra dimensions, as brane world models [35-39], string [40] and Kaluza-Klein theories [41], among others. Having adopted a particular model, the cosmological parameters can be determined by using statistical analysis of observational data.

However, some works have tried to explore the history of the universe without to appeal to any specific cosmological model. Such approaches are sometimes called cosmography or cosmokinetic models [42-47], and we will refer to them simply as kinematic models. This nomenclature comes from the fact that the complete study of the expansion of the Universe (or its kinematics) is described just by the Hubble expansion rate $H=\dot{a} / a$, the deceleration parameter $q=-a \ddot{a} / \dot{a}^{2}$ and the jerk parameter $j=-\dddot{a} a^{3} /\left(a \dot{a}^{3}\right)$, where $a$ is the scale factor in the Friedmann-Roberson-Walker (FRW) metric. The only assumption is that space-time is homogeneous and isotropic. In such parametrization, a simple dark matter dominated universe has $q=1 / 2$ while the accelerating $\Lambda$ CDM model has $j=-1$. The deceleration parameter allows to study the transition from a decelerated phase to an accelerated one, 
while the jerk parameter allows to study departures from the cosmic concordance model, without restricting to a specific model.

Concerning the deceleration parameter, several studies have attempted to estimate at which redshift $z_{\mathrm{t}}$ the universe undergoes a transition to accelerated phase $[18,19,44,48-$ $50,54,55]$. A model independent determination of the present deceleration parameter $q_{0}$ and deceleration-acceleration transition redshift $z_{\mathrm{t}}$ is of fundamental importance in modern cosmology. As a new cosmic parameter [54], it should be used to test several cosmological models.

In order to study the deceleration parameter in a cosmological-model independent framework, it is necessary to use some parametrization for it. This methodology has both advantages and disadvantages. One advantage is that it is independent of the matter and energy content of the universe. One disadvantage of this formulation is that it does not explain the cause of the accelerated expansion. Furthermore, the value of the present deceleration parameter may depend on the assumed form of $q(z)$. One of the first analyses and constraints on cosmological parameters of kinematic models was done by Elgarøy and Multamäki [46] by employing Bayesian marginal likelihood analysis. Since then, several authors have implemented the analysis by including new data sets and also different parametrizations for $q(z)$.

For a linear parametrization, $q(z)=q_{0}+q_{1} z$, the values for $q_{0}$ and $z_{\mathrm{t}}$ found by Cunha and Lima [48] were $q_{0} \sim-0.7, z_{\mathrm{t}}=0.43_{-0.05}^{+0.09}$ from $182 \mathrm{SNe}$ Ia of Riess et al. [4], $-1.17 \leq$ $q_{0} \leq-0.16, z_{\mathrm{t}}=0.61_{-0.21}^{+3.68}$ from SNLS data set [3] and $-1.0 \leq q_{0} \leq-0.36, z_{\mathrm{t}}=0.60_{-0.11}^{+0.28}$ from Davis et al. data set [5]. Guimarães, Cunha and Lima [49] found $q_{0}=-0.71 \pm 0.21$ and $z_{\mathrm{t}}=0.49_{-0.09}^{+0.27}$ using a sample of $307 \mathrm{SNe}$ Ia from Union compilation [6]. Also, for the linear parametrization, Rani et al. [50] found $q_{0}=-0.52 \pm 0.12$ and $z_{\mathrm{t}} \approx 0.98$ using a joint analysis of age of galaxies, strong gravitational lensing and SNe Ia data. For a parametrization of type $q(z)=q_{0}+q_{1} z /(1+z), \mathrm{Xu}, \mathrm{Li}$ and $\mathrm{Lu}[51]$ used $307 \mathrm{SNe}$ Ia together with $\mathrm{BAO}$ and $H(z)$ data and found $q_{0}=-0.715 \pm 0.045$ and $z_{\mathrm{t}}=0.609_{-0.070}^{+0.110}$. For the same parametrization, Holanda, Alcaniz and Carvalho [52] found $q_{0}=-0.85_{-1.25}^{+1.35}$ by using galaxy clusters of elliptical morphology based on their Sunyaev-Zeldovich effect (SZE) and X-ray observations. Such parametrization has also been studied in [46, 48].

As one may see, the determination of the deceleration parameter is a relevant subject in modern cosmology, as well as the determination of the Hubble parameter $H_{0}$. In seventies, Sandage [53] foretold that the determination of $H_{0}$ and $q_{0}$ would be the main role of cosmology for the forthcoming decades. The inclusion of the transition redshift $z_{\mathrm{t}}$ as a new cosmic discriminator has been advocated by some authors [54]. An alternative method to access the cosmological parameters in a model independent fashion is by means of the study of $H(z)=$ $\dot{a} / a=-[1 /(1+z)] d z / d t$. In the so called cosmic chronometer approach, the quantity $d z$ is obtained from spectroscopic surveys and the only quantity to be measured is the differential age evolution of the universe $(d t)$ in a given redshift interval $(d z)$. By using the results from Baryon Oscillation Spectroscopic Survey (BOSS) [14-16], Moresco et al. [55] have obtained a cosmological-model independent determination of the transition redshift as $z_{\mathrm{t}}=0.4 \pm 0.1$ (see also [17-19]).

In the present work we study the transition redshift by means of a third order parametrization of the comoving distance, a second order parametrization of $H(z)$ and a linear parametrization of $q(z)$. By combining luminosity distances from $\mathrm{SNe}$ Ia [56] and $H(z)$ measurements, it is possible to determine $z_{\mathrm{t}}$ values in these cosmological-model independent frameworks. In such approach we obtain an interesting complementarity between the observational data and, consequently, tighter constraints on the parameter spaces. 
The paper is organized as follows. In section 2, we present the basic equations concerning the obtainment of $z_{\mathrm{t}}$ from luminosity distance, $H(z)$ and $q(z)$. Section 3 presents the data set used and the analyses are presented in section 4 . Conclusions are left to section 5 .

\section{Basic equations}

Let us discuss (from a more observational viewpoint) the possibility to enlarge Sandage's vision by including the transition redshift, $z_{\mathrm{t}}$, as the third cosmological number. To begin with, consider the general expression for the deceleration parameter $q(z)$ as given by:

$$
q(z)=-\frac{\ddot{a}}{a H^{2}}=\frac{1+z}{H} \frac{d H}{d z}-1,
$$

from which the transition redshift, $z_{\mathrm{t}}$, can be defined as $q\left(z_{\mathrm{t}}\right)=0$, leading to:

$$
z_{\mathrm{t}}=\left[\frac{d \ln H(z)}{d z}\right]_{\left.\right|_{z=z_{\mathrm{t}}}}^{-1}-1 .
$$

Let us assume a flat Friedmann-Robertson-Walker cosmology. In such a framework, the luminosity distance, $d_{\mathrm{L}}$ (in $\mathrm{Mpc}$ ), is given by:

$$
d_{\mathrm{L}}(z)=(1+z) d_{\mathrm{C}}(z),
$$

where $d_{\mathrm{C}}$ is the comoving distance:

$$
d_{\mathrm{C}}(z)=c \int_{0}^{z} \frac{d z^{\prime}}{H\left(z^{\prime}\right)}
$$

with $c$ being the speed of light in $\mathrm{km} / \mathrm{s}$ and $H(z)$ the Hubble parameter in $\mathrm{km} / \mathrm{s} / \mathrm{Mpc}$. For mathematical convenience, we choose to work with dimensionless quantities. Then, we define the dimensionless distances, $D_{\mathrm{C}} \equiv \frac{d_{\mathrm{C}}}{d_{H}}, D_{\mathrm{L}} \equiv \frac{d_{\mathrm{L}}}{d_{H}}, d_{H} \equiv c / H_{0}$ and the dimensionless Hubble parameter, $E(z) \equiv \frac{H(z)}{H_{0}}$. Thus, we have:

$$
D_{\mathrm{L}}(z)=(1+z) D_{\mathrm{C}}(z)
$$

and

$$
D_{\mathrm{C}}(z)=\int_{0}^{z} \frac{d z^{\prime}}{E\left(z^{\prime}\right)}
$$

from which follows

$$
E(z)=\left[\frac{d D_{\mathrm{C}}(z)}{d z}\right]^{-1}
$$

From (2.2) we also have:

$$
z_{\mathrm{t}}=\left[\frac{d \ln E(z)}{d z}\right]_{\mid z=z_{\mathrm{t}}}^{-1}-1
$$

Therefore, from a formal point of view, we may access the value of $z_{\mathrm{t}}$ through a parametrization of both $q(z)$ and $H(z)$, at least around a redshift interval involving the transition redshift. As a third method we can also parametrize the co-moving distance, which is directly related to the luminosity distance, in order to study the transition redshift. In which follows we present the three different methods considered here. 


\section{$2.1 \quad z_{\mathrm{t}}$ from comoving distance, $D_{\mathrm{C}}(z)$}

In order to put limits on $z_{\mathrm{t}}$ by considering the comoving distance, we can write $D_{\mathrm{C}}(z)$ by a third degree polynomial such as:

$$
D_{\mathrm{C}}=z+d_{2} z^{2}+d_{3} z^{3} .
$$

where $d_{2}$ and $d_{3}$ are free parameters. Naturally, from eqs. (2.7) and (2.9), one obtains

$$
E(z)=\frac{1}{1+2 d_{2} z+3 d_{3} z^{2}} .
$$

Solving eq. (2.8) with $E(z)$ given by (2.10), we find

$$
z_{\mathrm{t}}=\frac{-2 d_{2}-3 d_{3} \pm \sqrt{4 d_{2}^{2}-6 d_{2} d_{3}+9 d_{3}^{2}-9 d_{3}}}{9 d_{3}}
$$

where we may see there are two possible solutions to $z_{\mathrm{t}}$. From a statistical point of view, aiming to constrain $z_{\mathrm{t}}$, maybe it is better to write the coefficient $d_{3}$ in terms of $z_{\mathrm{t}}$ and $d_{2}$ via eq. (2.8) as

$$
d_{3}\left(d_{2}, z_{\mathrm{t}}\right)=-\frac{1+2 d_{2}+4 d_{2} z_{\mathrm{t}}}{3 z_{\mathrm{t}}\left(2+3 z_{\mathrm{t}}\right)}
$$

Then

$$
E(z)=\left[1+2 d_{2} z-\frac{1+2 d_{2}+4 d_{2} z_{\mathrm{t}}}{z_{\mathrm{t}}\left(2+3 z_{\mathrm{t}}\right)} z^{2}\right]^{-1} .
$$

Finally, from eqs. (2.5), (2.9) and (2.12) the dimensionless luminosity distance is

$$
D_{\mathrm{L}}(z)=(1+z)\left[z+d_{2} z^{2}-\frac{1+2 d_{2}+4 d_{2} z_{\mathrm{t}}}{3 z_{\mathrm{t}}\left(2+3 z_{\mathrm{t}}\right)} z^{3}\right] .
$$

Equations (2.14) and (2.13) are to be compared with luminosity distances from SNe Ia and $H(z)$ measurements, respectively, in order to determine $z_{\mathrm{t}}$ and $d_{2}$.

\section{$2.2 z_{\mathrm{t}}$ from $H(z)$}

In order to assess $z_{\mathrm{t}}$ from eq. (2.2) by means of $H(z)$ we need an expression for $H(z)$. If one wants to avoid dynamical assumptions, one must to resort to kinematical methods which uses an expansion of $H(z)$ over the redshift.

The simplest expansion of $H(z)$ over the redshift, the linear expansion, gives no transition. To realize this, let us take

$$
\frac{H(z)}{H_{0}}=E(z)=1+h_{1} z
$$

From (2.8), we have

$$
1+z_{\mathrm{t}}=\left[\frac{d \ln E(z)}{d z}\right]_{z=z_{\mathrm{t}}}^{-1}=\frac{1}{h_{1}}+z_{\mathrm{t}} \Rightarrow \frac{1}{h_{1}}=1 .
$$

Therefore, the transition redshift is undefined in this case. 
Let us now try the next simplest $H(z)$ expansion, namely, the quadratic expansion:

$$
\frac{H(z)}{H_{0}}=E(z)=1+h_{1} z+h_{2} z^{2} .
$$

In this case, inserting (2.10) into (2.16), we are left with:

$$
1+z_{\mathrm{t}}=\frac{1+h_{1} z_{\mathrm{t}}+h_{2} z_{\mathrm{t}}^{2}}{h_{1}+2 h_{2} z_{\mathrm{t}}} \Rightarrow\left(1+z_{\mathrm{t}}\right)\left(h_{1}+2 h_{2} z_{\mathrm{t}}\right)=1+h_{1} z_{\mathrm{t}}+h_{2} z_{\mathrm{t}}^{2},
$$

from which follows an equation for $z_{\mathrm{t}}$ :

$$
h_{2} z_{\mathrm{t}}^{2}+2 h_{2} z_{\mathrm{t}}+h_{1}-1=0
$$

whose solution is:

$$
z_{\mathrm{t}}=-1 \pm \sqrt{1+\frac{1-h_{1}}{h_{2}}} .
$$

We may exclude the negative root, which would give $z_{\mathrm{t}}<-1$ and this value is not possible (negative scale factor). Thus, if one obtains the $h_{1}$ and $h_{2}$ coefficients from a fit to $H(z)$ data, one may obtain a model independent estimate of transition redshift from

$$
z_{\mathrm{t}}=-1+\sqrt{1+\frac{1-h_{1}}{h_{2}}} .
$$

Equation (2.21) already is an interesting result, and shows the reliability of the quadratic model as a kinematic assessment of transition redshift. It is easy to see that taking $h_{2}=0$ into (2.19) does not furnish any information about the transition redshift.

In order to constrain the model with $\mathrm{SNe}$ Ia data, we obtain the luminosity distance from eqs. (2.5), (2.6) and (2.17). We have

$$
D_{\mathrm{C}}=\int_{0}^{z} \frac{d z^{\prime}}{E\left(z^{\prime}\right)}=\int_{0}^{z} \frac{d z^{\prime}}{1+h_{1} z^{\prime}+h_{2} z^{\prime 2}}
$$

which gives three possible solutions, according to the sign of $\Delta \equiv h_{1}^{2}-4 h_{2}$ such as

$$
D_{\mathrm{C}}= \begin{cases}\frac{2}{\sqrt{-\Delta}}\left[\arctan \left(\frac{2 h_{2} z+h_{1}}{\sqrt{-\Delta}}\right)-\arctan \frac{h_{1}}{\sqrt{-\Delta}}\right], & \Delta<0 \\ \frac{2 z}{h_{1} z+2}, & \Delta=0 \\ \frac{1}{\sqrt{\Delta}} \ln \left|\left(\frac{\sqrt{\Delta}+h_{1}}{\sqrt{\Delta}-h_{1}}\right)\left(\frac{\sqrt{\Delta}-h_{1}-2 h_{2} z}{\sqrt{\Delta}+h_{1}+2 h_{2} z}\right)\right|, & \Delta>0 .\end{cases}
$$

However, in order to obtain the likelihood for the transition redshift, we must reparametrize the eq. (2.17) to show its explicit dependency on this parameter. Notice also that from eq. (2.21) we may eliminate the parameter $h_{1}$ :

$$
h_{1}=1+h_{2}\left[1-\left(1+z_{\mathrm{t}}\right)^{2}\right]
$$

thus we may write $D_{\mathrm{C}}(z)$ from (2.23) just in terms of $z_{\mathrm{t}}$ and $h_{2}$, from which follows the luminosity distance $D_{\mathrm{L}}=D_{\mathrm{C}}(z)(1+z)$. 


\section{$2.3 \quad z_{\mathrm{t}}$ from $q(z)$}

Now let us see how to assess $z_{\mathrm{t}}$ by means of a parametrization of $q(z)$. From (2.1) one may find $E(z)$

$$
E(z)=\exp \left[\int_{0}^{z} \frac{1+q\left(z^{\prime}\right)}{1+z^{\prime}} d z^{\prime}\right]
$$

If we assume a linear $z$ dependence in $q(z)$, as

$$
q(z)=q_{0}+q_{1} z
$$

which is the simplest $q(z)$ parametrization that allows for a transition, one may find

$$
E(z)=e^{q_{1} z}(1+z)^{1+q_{0}-q_{1}}
$$

while the comoving distance $D_{\mathrm{C}}(z)(2.6)$ is given by

$$
D_{\mathrm{C}}(z)=e^{q_{1}} q_{1}^{q_{0}-q_{1}}\left[\Gamma\left(q_{1}-q_{0}, q_{1}\right)-\Gamma\left(q_{1}-q_{0}, q_{1}(1+z)\right)\right]
$$

where $\Gamma(a, x)$ is the incomplete gamma function defined by [57] as $\Gamma(a, x) \equiv \int_{x}^{\infty} e^{-t} t^{a-1} d t$, with $a>0$.

From (2.26) (or from (2.27) and (2.8)) it is easy to find:

$$
z_{\mathrm{t}}=-\frac{q_{0}}{q_{1}} \quad \text { and } \quad q_{0}=-q_{1} z_{\mathrm{t}}
$$

from which follows $D_{\mathrm{C}}(z)$ and $D_{\mathrm{L}}(z)$ as a function of just $z_{\mathrm{t}}$ and $q_{1}$, which can be constrained from observational data.

\section{Samples}

\section{$3.1 \quad H(z)$ data}

Hubble parameter data in terms of redshift yields one of the most straightforward cosmological tests because it is inferred from astrophysical observations alone, not depending on any background cosmological models.

At the present time, the most important methods for obtaining $H(z)$ data are $^{1}$ (i) through "cosmic chronometers", for example, the differential age of galaxies (DAG), (ii) measurements of peaks of acoustic oscillations of baryons (BAO) and (iii) through correlation function of luminous red galaxies (LRG).

The data we work here are a combination of the compilations from Sharov and Vorontsova [58] and Moresco et al. [55] as described on Jesus et al. [59]. Sharov and Vorontsova [58] added $6 H(z)$ data in comparison to Farooq and Ratra [18] compilation, which had 28 measurements. Moresco et al. [55], on their turn, have added 7 new $H(z)$ measurements in comparison to Sharov and Vorontsova [58]. By combining both datasets, Jesus et al. [59] have arrived at $41 H(z)$ data, as can be seen on table 1 of [59] and figure 1b here.

\footnotetext{
${ }^{1}$ See [54] for a review.
} 


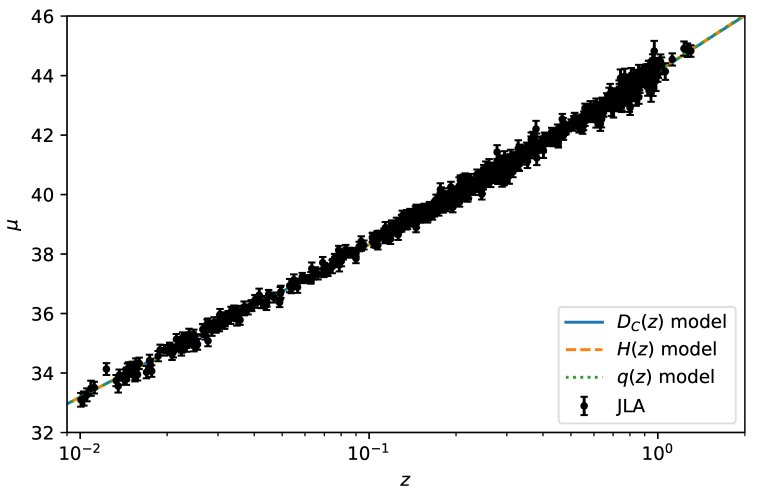

(a)

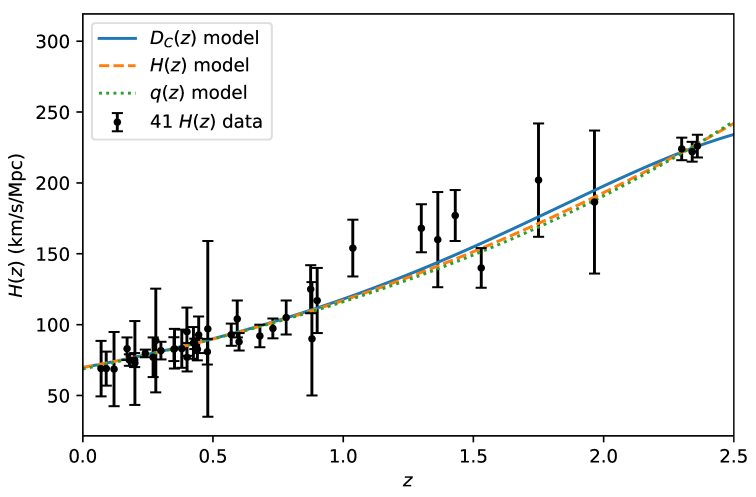

(b)

Figure 1. a) SNe Ia distance moduli from JLA. The data for $\mu$ were estimated from eq. (4.2), with SNe Ia parameters from the $D_{\mathrm{C}}(z)$ model (table 1) and the error bars comes from the diagonal of the covariance matrix. The lines represent the best fit from $\mathrm{SNe}+H(z)$ data for each model. b) $41 H(z)$ data compilation. The lines represent the best fit from $\mathrm{SNe}+H(z)$ data for each model.

\subsection{JLA SNe Ia compilation}

The JLA compilation [56] consists of 740 SNe Ia from the SDSS-II [60] and SNLS [61] collaborations. Actually, this compilation produced recalibrated SNe Ia light-curves and associated distances for the SDSS-II and SNLS samples in order to improve the accuracy of cosmological constraints, limited by systematic measurement uncertainties, as, for instance, the uncertainty in the band-to-band and survey-to-survey relative flux calibration. The light curves have high quality and were obtained by using an improved SALT2 (Spectral Adaptive Light-curve Templates) method [56, 62-64]. The data set includes several lowredshift samples $(z<0.1)$, all three seasons from the SDSS-II $(0.05 \leq z \leq 0.4)$ and three years from SNLS $(0.2<z<1.4)$. See figure 1a and more details in next section.

\section{Analyses and results}

In our analyses, we have used flat priors over the parameters, so the posteriors are always proportional to the likelihoods. For $H(z)$ data, the likelihood distribution function is given by $\mathscr{L}_{H} \propto e^{-\frac{\chi_{H}^{2}}{2}}$, where

$$
\chi_{H}^{2}=\sum_{i=1}^{41} \frac{\left[H_{\mathrm{obs}, i}-H\left(z_{i}, H_{0}, z_{\mathrm{t}}, \theta_{\mathrm{mod}, j}\right)\right]^{2}}{\sigma_{H_{i}, \mathrm{obs}}^{2}},
$$

where $\theta_{\text {mod, } j}$ is the specific parameter for each model, namely $d_{2}, h_{2}$ or $q_{1}$, for $D_{\mathrm{C}}(z), H(z)$, $q(z)$ parametrizations, respectively.

As explained on [56], we may assume that supernovae with identical color, shape and galactic environment have, on average, the same intrinsic luminosity for all redshifts. In this case, the distance modulus $\mu=5 \log _{10}\left(d_{\mathrm{L}}(\mathrm{pc}) / 10\right)$ may be given by

$$
\mu=m_{B}^{*}-\left(M_{B}-\alpha \times X_{1}+\beta \times C\right)
$$

where $X_{1}$ describes the time stretching of the light-curve, $C$ describes the supernova color at maximum brightness, $m_{B}^{*}$ corresponds to the observed peak magnitude in the rest-frame 
$B$ band, $\alpha, \beta$ and $M_{B}$ are nuisance parameters. According to [61], $M_{B}$ may depend on the host stellar mass $\left(M_{\text {stellar }}\right)$ as

$$
M_{B}= \begin{cases}M_{B}^{1} & \text { if } M_{\text {stellar }}<10^{10} M_{\odot} . \\ M_{B}^{1}+\Delta_{M} & \text { otherwise }\end{cases}
$$

For SNe Ia from JLA, we have the likelihood $\mathscr{L}_{S N} \propto e^{-\frac{\chi_{S N}^{2}}{2}}$, where

$$
\chi_{S N}^{2}=\left[\hat{\boldsymbol{\mu}}\left(\theta_{S N}\right)-\boldsymbol{\mu}\left(z, z_{\mathrm{t}}, \theta_{\bmod , j}\right)\right]^{T} \boldsymbol{C}^{-1}\left[\hat{\boldsymbol{\mu}}\left(\theta_{S N}\right)-\boldsymbol{\mu}\left(z, z_{\mathrm{t}}, \theta_{\bmod , j}\right)\right]
$$

where $\theta_{S N}=\left(\alpha, \beta, M_{B}^{1}, \Delta_{M}\right), \boldsymbol{C}$ is the covariance matrix of $\hat{\boldsymbol{\mu}}$ as described on [56], $\boldsymbol{\mu}\left(z, z_{\mathrm{t}}\right.$, $\left.\theta_{\text {mod }, j}\right)=5 \log _{10}\left(d_{\mathrm{L}}\left(z, z_{\mathrm{t}}, \theta_{\text {mod }, j}\right) / 10 \mathrm{pc}\right)$ computed for a fiducial value $H_{0}=70 \mathrm{~km} / \mathrm{s} / \mathrm{Mpc}$.

In order to obtain the constraints over the free parameters, we have sampled the likelihood $\mathscr{L} \propto e^{-\chi^{2} / 2}$ through Monte Carlo Markov Chain (MCMC) analysis. A simple and powerful MCMC method is the so-called Affine Invariant MCMC Ensemble Sampler by [65], which was implemented in Python language with the emcee software by [66]. This MCMC method has advantage over the simple Metropolis-Hastings (MH) method, since it depends only on one scale parameter of the proposed distribution and also on the number of walkers, while MH method is based on the parameter covariance matrix, that is, it depends on $n(n+1) / 2$ tuning parameters, where $n$ is the dimension of parameter space. The main idea of the Goodman-Weare affine-invariant sampler is the so called "stretch move", where the position (parameter vector in parameter space) of a walker (chain) is determined by the position of the other walkers. Foreman-Mackey et al. modified this method, in order to make it suitable for parallelization, by splitting the walkers in two groups, then the position of a walker in one group is determined only by the position of walkers of the other group. ${ }^{2}$

We used the freely available software emcee to sample from our likelihood in $n$ dimensional parameter space. We have used flat priors over the parameters. In order to plot all the constraints on each model in the same figure, we have used the freely available software getdist, ${ }^{3}$ in its Python version. The results of our statistical analyses can be seen on figures $2-8$ and on table 1 .

In figures 2-4, we have the combined results for each parametrization. As one may see, we have always chosen $z_{\mathrm{t}}$ as one of our fiducial parameter. The other parameters from each model is later obtained as derived parameters. As one may see in figures $2-4$, the combination of JLA $+H(z)$ yields strong constraints over all parameters, especially $z_{\mathrm{t}}$. Also, we find negligible difference in the SNe Ia parameters for each model. We have also to emphasize that the constraints over $H_{0}$ comes just from $H(z)$ while for JLA $H_{0}$ is fixed. We choose not to include other constraints over $H_{0}$ due to the recent tension from different limits over the Hubble constant. At the end of this section, we compare our results with different constraints over $H_{0}$.

In figures $5-7$, we show explicitly the independent constraints from JLA and $H(z)$ over the cosmological parameters. As one may see in figure 5 , SNe Ia sets weaker constraints over $z_{\mathrm{t}}$ for $D_{\mathrm{C}}(z)$ parametrization. Almost all the $z_{\mathrm{t}}$ constraint comes just from $H(z)$. For $d_{2}$, $H(z)$ and JLA yields similar constraints. For $d_{3}, H(z)$ yields slightly better constraints. For figures 6 and 7, the constraints over $z_{\mathrm{t}}$ from SNe Ia are improved and one may see how SNe Ia and $H(z)$ complement each other in order to constrain the transition redshift. In figure 6,

\footnotetext{
${ }^{2}$ See [67] for a comparison among various MCMC sampling techniques.

${ }^{3}$ getdist is part of the great MCMC sampler and CMB power spectrum solver COSMOMC, by [68].
} 


\begin{tabular}{|l|c|c|c|}
\hline Parameter & $D_{\mathrm{C}}(z)$ & $H(z)$ & $q(z)$ \\
\hline $\boldsymbol{\alpha}$ & $0.1412 \pm 0.0065 \pm 0.013$ & $0.1412 \pm 0.0066 \pm 0.013$ & $0.1408 \pm 0.0064 \pm 0.013$ \\
$\boldsymbol{\beta}$ & $3.105 \pm 0.080 \pm 0.16$ & $3.101 \pm 0.082 \pm 0.16$ & $3.094 \pm 0.080 \pm 0.16$ \\
$\boldsymbol{M}_{\boldsymbol{B}}^{\mathbf{1}}$ & $-19.073 \pm 0.044_{-0.090}^{+0.089}$ & $-19.039 \pm 0.023_{-0.045}^{+0.047}$ & $-19.033 \pm 0.023_{-0.046}^{+0.045}$ \\
$\boldsymbol{\Delta}_{\boldsymbol{M}}$ & $-0.069 \pm 0.023 \pm 0.046$ & $-0.071 \pm 0.023_{-0.046}^{+0.045}$ & $-0.071 \pm 0.023_{-0.047}^{+0.046}$ \\
$\boldsymbol{H}_{\mathbf{0}}$ & $69.1 \pm 1.5 \pm 3.0$ & $68.8 \pm 1.6 \pm 3.2$ & $68.6 \pm 1.6_{-3.2}^{+3.3}$ \\
$\boldsymbol{z}_{\mathbf{t}}$ & $0.806 \pm 0.094_{-0.18}^{+0.19}$ & $0.870 \pm 0.063_{-0.12}^{+0.13}$ & $0.973 \pm 0.058_{-0.11}^{+0.12}$ \\
$\boldsymbol{d}_{\mathbf{2}}$ & $-0.253 \pm 0.016_{-0.031}^{+0.033}$ & - & - \\
$d_{3}$ & $0.0299 \pm 0.0044_{-0.0090}^{+0.0085}$ & - & - \\
$h_{1}$ & - & $0.522 \pm 0.065 \pm 0.13$ & - \\
$\boldsymbol{h}_{\mathbf{2}}$ & - & $0.192 \pm 0.026_{-0.052}^{+0.051}$ & - \\
$q_{0}$ & - & - & $-0.434 \pm 0.065 \pm 0.13$ \\
$\boldsymbol{q}_{\mathbf{1}}$ & - & - & $0.446 \pm 0.062 \pm 0.12$ \\
\hline
\end{tabular}

Table 1. Constraints from JLA $+H(z)$ for $D_{\mathrm{C}}(z), H(z)$ and $q(z)$ parametrizations. The parameters without bold faces were treated as derived parameters. The central values correspond to the mean and the $1 \sigma$ and $2 \sigma$ c.l. correspond to the minimal $68.3 \%$ and $95.4 \%$ confidence intervals.

one may see that the constraint over $h_{1}$ is better from JLA. The constraint over $h_{2}$ is better from $H(z)$. In figure 7 , one may see that the constraint over $q_{0}$ is better from JLA. The constraint over $q_{1}$ is better from $H(z)$.

For all parametrizations, the best constraints over the transition redshift comes from $H(z)$ data, as first indicated by [54]. Moresco et al. [55] also found stringent constraints over $z_{\mathrm{t}}$ from $H(z)$ in their parametrization, however, they did not compare with SNe Ia constraints.

Figure 8 summarizes our combined constraints over $z_{\mathrm{t}}$ for each parametrization. As one may see, the $q(z)$ model yields the strongest constraints over $z_{\mathrm{t}}$. The other parametrizations are important for us to realize how much $z_{\mathrm{t}}$ is still allowed to vary. All constraints are compatible at $1 \sigma$ c.l.

Table 1 shows the full numerical results from our statistical analysis. As one may see the $\mathrm{SNe}$ Ia constraints vary little for each parametrization. In fact, we also have found constraints from the (faster) JLA binned data [56], however, when comparing with the (slower) full JLA constraints, we have found that the full JLA yields stronger constraints over the parameters, especially $z_{\mathrm{t}}$. So we decided to deal only with the JLA full data.

By using $30 H(z)$ data, plus $H_{0}$ from Riess et al. (2011) [69], Moresco et al. [55] found $z_{\mathrm{t}}=0.64_{-0.06}^{+0.1}$ for $\Lambda \mathrm{CDM}$ and $z_{\mathrm{t}}=0.4 \pm 0.1$ for their model independent approach. Only our $D_{\mathrm{C}}(z)$ parametrization is compatible with their model-independent result. Their $\Lambda$ CDM result is compatible with all our parametrizations, although it is marginally compatible with $q(z)$.

Another interesting result that can be seen in table 1 is the $H_{0}$ constraint. As one may see, the constraints over $H_{0}$ are consistent through the three different parametrizations, 


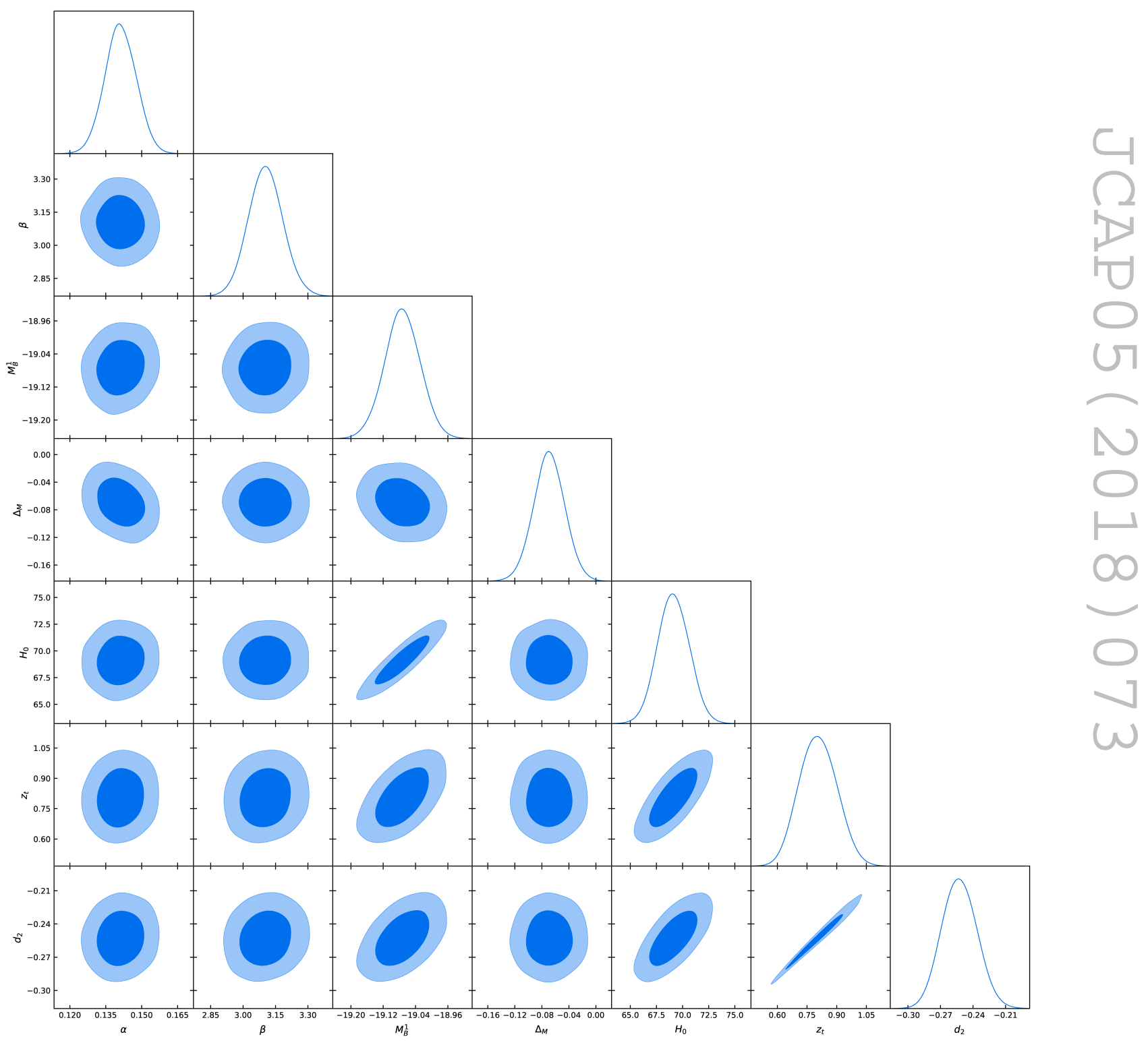

Figure 2. Combined constraints from JLA and $H(z)$ for $D_{\mathrm{C}}(z)=z+d_{2} z^{2}+d_{3} z^{3}$. 


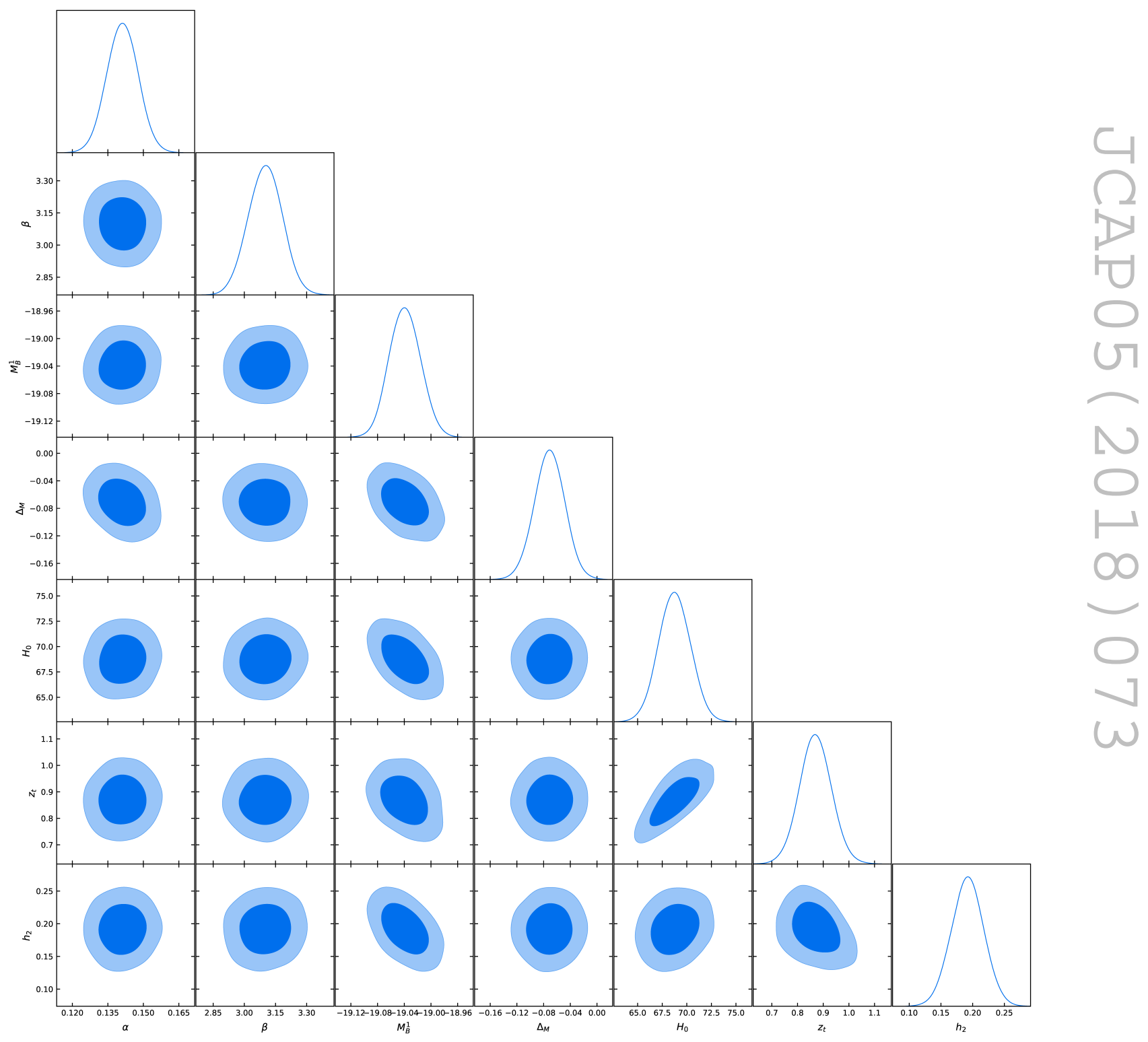

Figure 3. Combined constraints from JLA and $H(z)$ for $H(z)=H_{0}\left(1+h_{1} z+h_{2} z^{2}\right)$. 


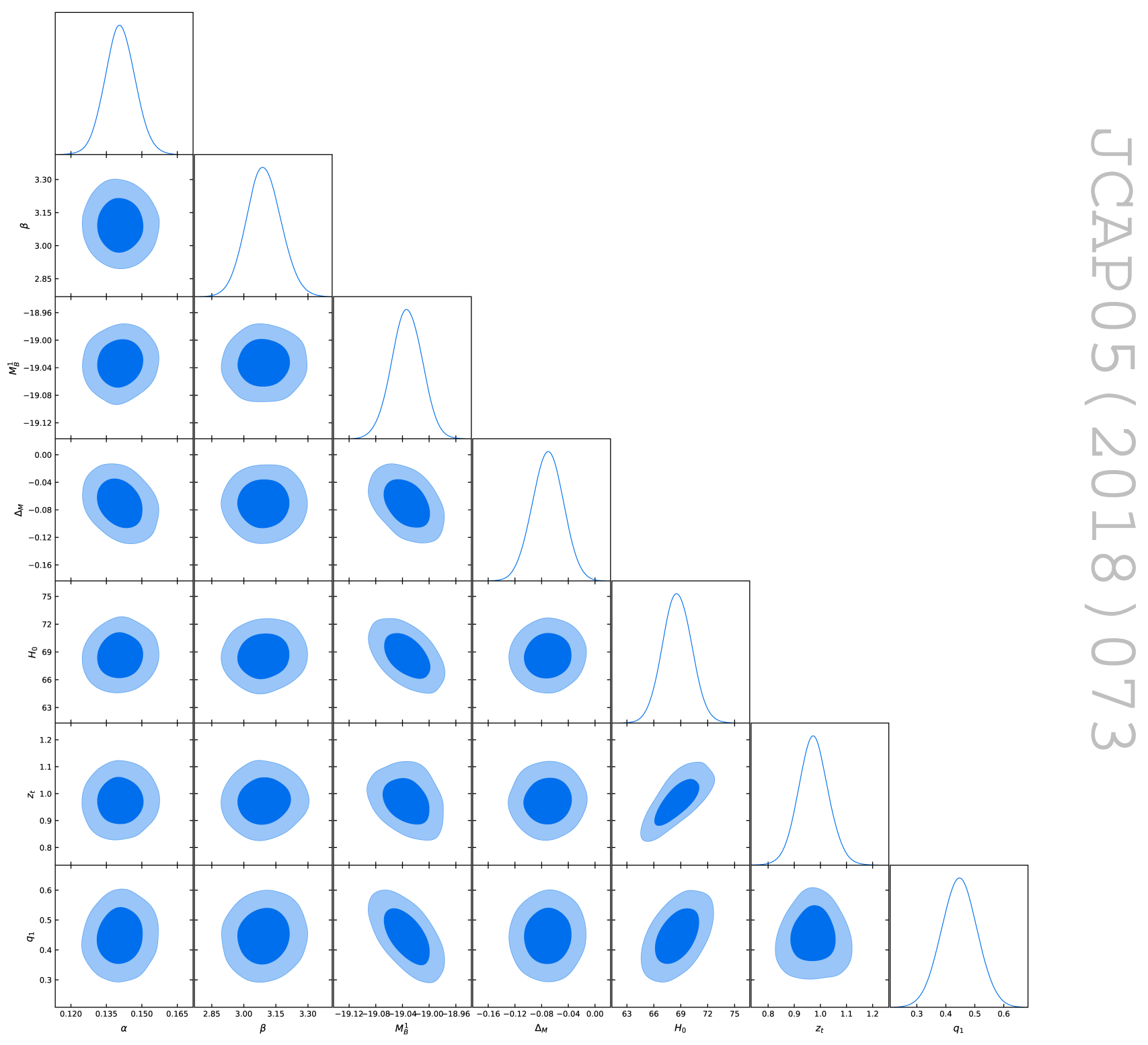

Figure 4. Combined constraints from JLA and $H(z)$ for $q(z)=q_{0}+q_{1} z$. 


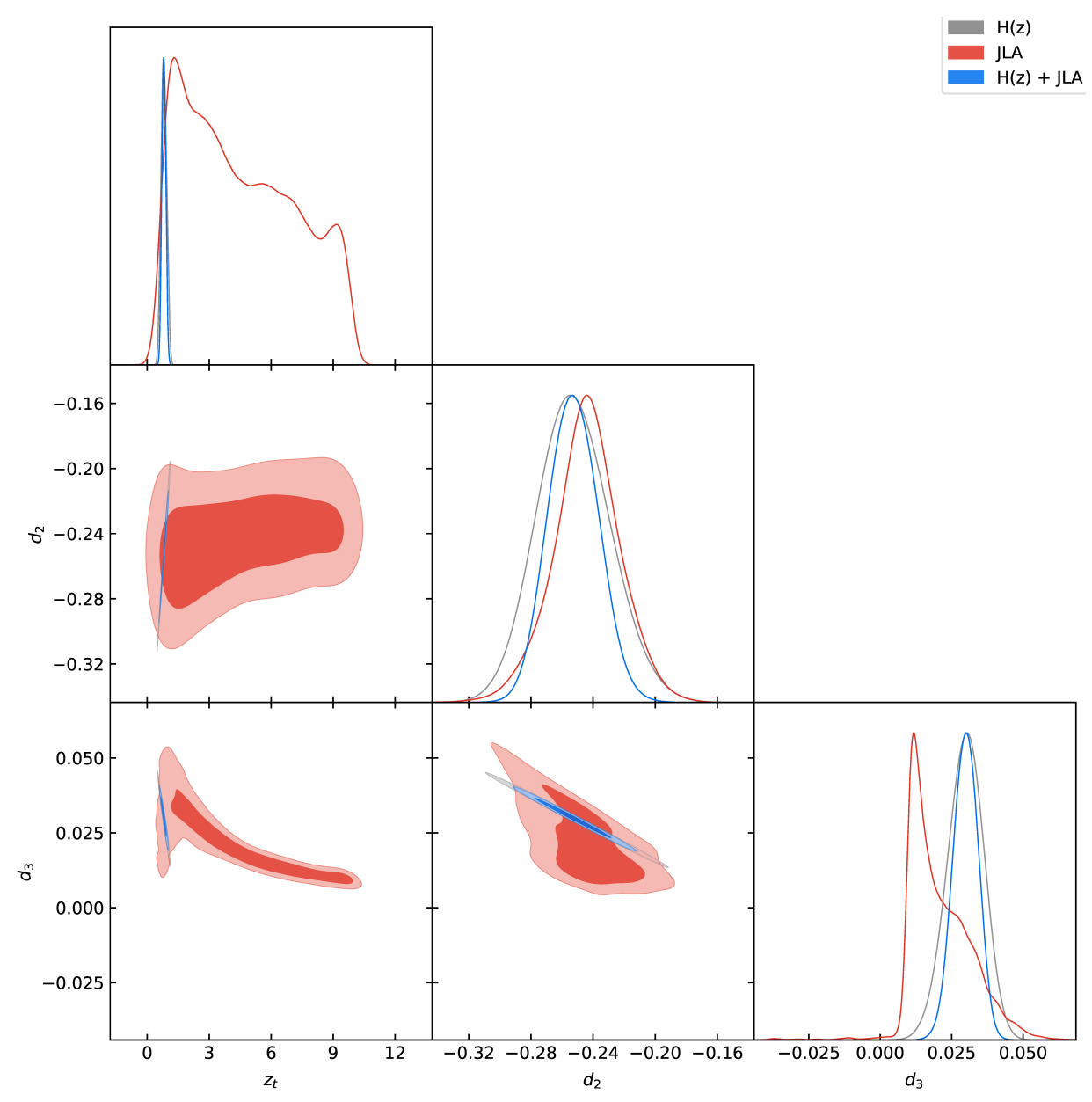

Figure 5. Constraints from JLA and $H(z)$ for $D_{\mathrm{C}}(z)=z+d_{2} z^{2}+d_{3} z^{3}$. 


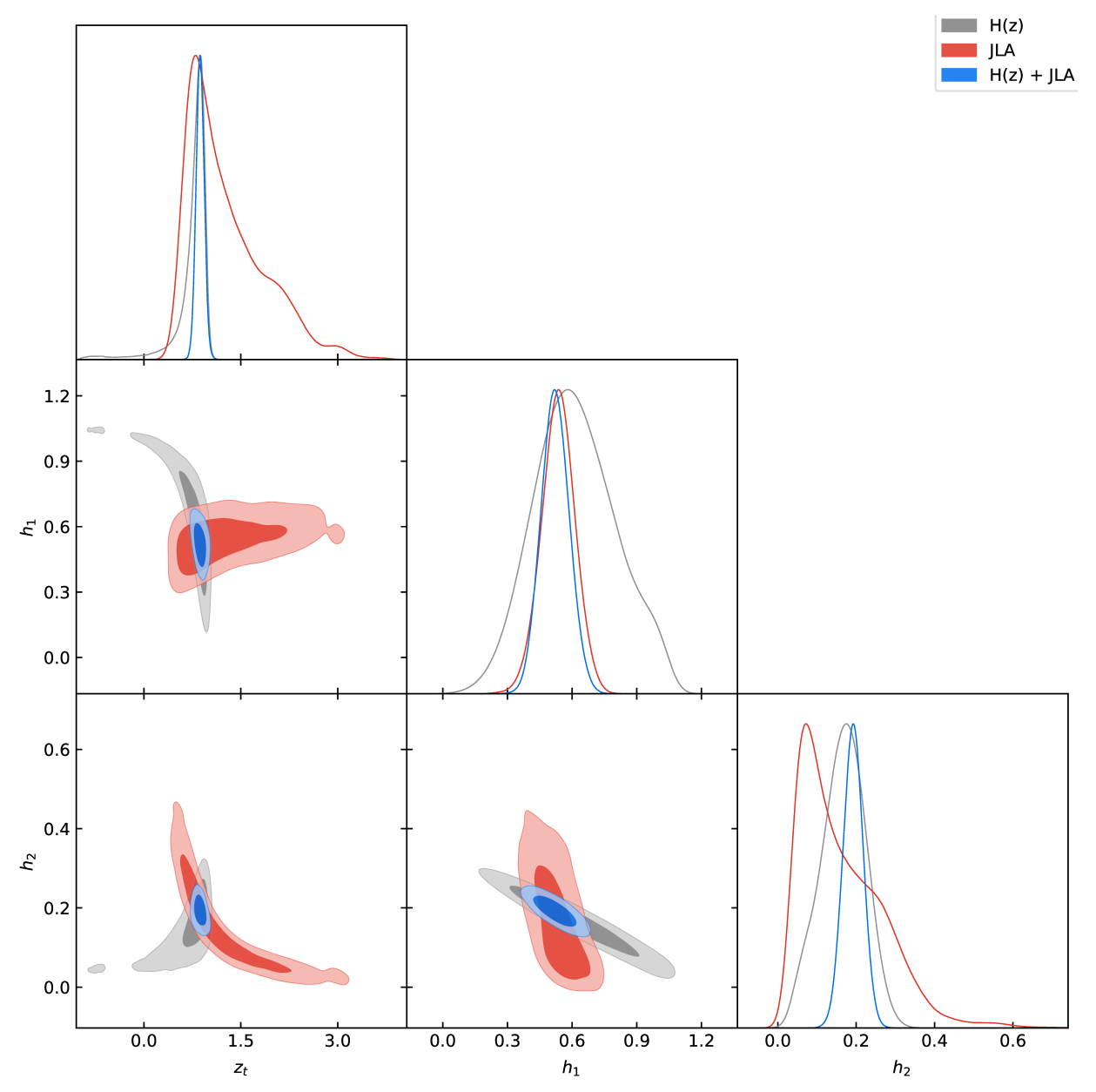

Figure 6. Constraints from JLA and $H(z)$ for $H(z)=H_{0}\left(1+h_{1} z+h_{2} z^{2}\right)$. 


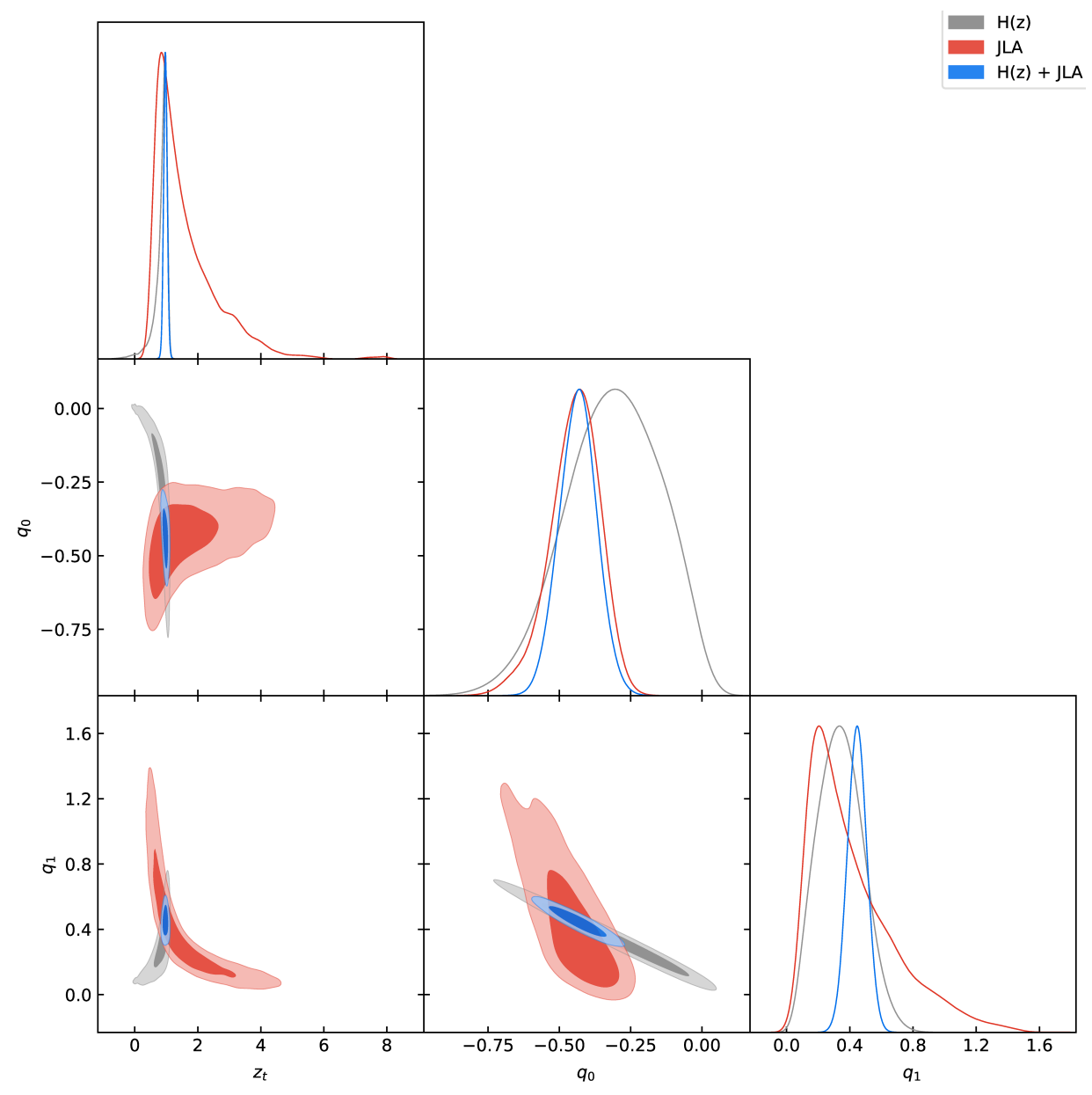

Figure 7. Constraints from JLA and $H(z)$ for $q(z)=q_{0}+q_{1} z$. 


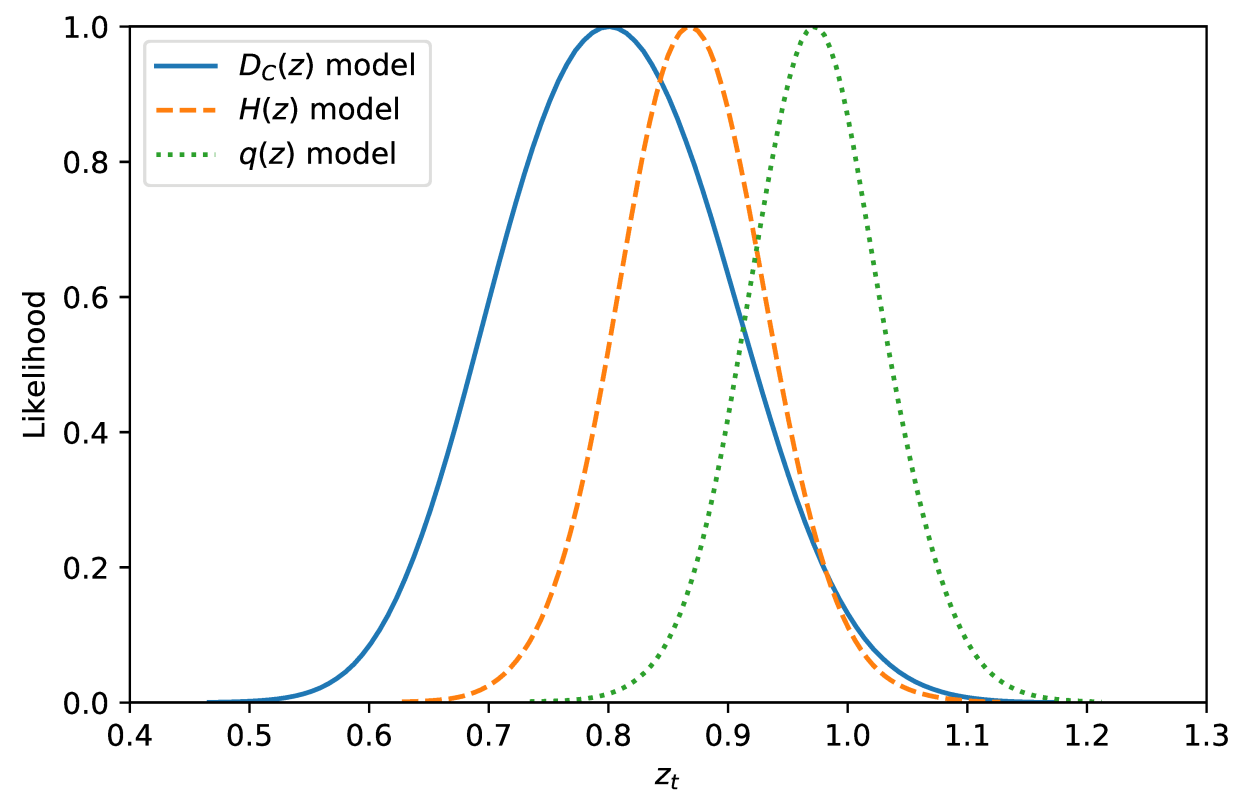

Figure 8. Likelihoods for transition redshift from JLA and $H(z)$ data combined. Blue solid line corresponds to $D_{\mathrm{C}}(z)$ parametrization, orange long-dashed line corresponds to $H(z)$ parametrization and green short-dashed line corresponds to $q(z)$ parametrization.

with a little smaller uncertainty for $D_{\mathrm{C}}(z), H_{0}=69.1 \pm 1.5 \mathrm{~km} / \mathrm{s} / \mathrm{Mpc}$. The constraints over $H_{0}$ are quite stringent today from many observations [70, 71]. However, there is some tension among $H_{0}$ values estimated from Cepheids [70] and from CMB [71]. While Riess et al. advocate $H_{0}=73.24 \pm 1.74 \mathrm{~km} / \mathrm{s} / \mathrm{Mpc}$, the Planck collaboration analysis yields $H_{0}=$ $66.93 \pm 0.62 \mathrm{~km} / \mathrm{s} / \mathrm{Mpc}$, a $3.4 \sigma$ lower value. It is interesting to note, from our table 1 that, although we are working with model independent parametrizations and data at intermediate redshifts, our result is in better agreement with the high redshift result from Planck. In fact, all our results are compatible within $1 \sigma$ with the Planck's result, while it is incompatible at $3 \sigma$ with the Riess' result.

\section{Conclusion}

The accelerated expansion of Universe is confirmed by different sets of cosmological observations. Several models proposed in literature satisfactorily explain the transition from decelerated phase to the current accelerated phase. A more significant question is when the transition occurs from one phase to another, and the parameter that measures this transition is called the transition redshift, $z_{\mathrm{t}}$. The determination of $z_{\mathrm{t}}$ is strongly dependent on the cosmological model adopted, thus the search for methods that allow the determination of such parameter in a model independent way are of fundamental importance, since it would serve as a test for several cosmological models.

In the present work, we wrote the comoving distance $D_{\mathrm{C}}$, the Hubble parameter $H(z)$ and the deceleration parameter $q(z)$ as third, second and first degree polynomials on $z$, respectively (see equations (2.9), (2.17) and (2.26)), and obtained, for each case, the $z_{\mathrm{t}}$ value. Only a flat universe was assumed and the estimates for $z_{\mathrm{t}}$ were obtained, independent of a specific cosmological model. As observational data, we have used Supernovae type Ia and 
Hubble parameter measurements. Our results can be found in figures 2-7. As one may see from figures $5-7$, the analyses by using $\mathrm{SNe}$ Ia (red color) and $H(z)$ data (blue color) are complementary to each other, providing tight limits in the parameter spaces. As a result, the values obtained for the transition redshift in each case were $0.806 \pm 0.094,0.870 \pm 0.063$ and $0.973 \pm 0.058$ at $1 \sigma$ c.l., respectively (see figure 8).

\section{Acknowledgments}

JFJ is supported by Fundação de Amparo à Pesquisa do Estado de São Paulo - FAPESP (Process no. 2017/05859-0). RFLH acknowledges financial support from Conselho Nacional de Desenvolvimento Científico e Tecnológico (CNPq) and UEPB (No. 478524/2013-7, 303734/2014-0). SHP is grateful to CNPq, for financial support (No. 304297/2015-1, 400924/2016-1).

\section{References}

[1] Supernova Search Team collaboration, A.G. Riess et al., Observational evidence from supernovae for an accelerating universe and a cosmological constant, Astron. J. 116 (1998) 1009 [astro-ph/9805201] [INSPIRE].

[2] Supernova Cosmology Project collaboration, S. Perlmutter et al., Measurements of $\Omega$ and $\Lambda$ from 42 high redshift supernovae, Astrophys. J. 517 (1999) 565 [astro-ph/9812133] [INSPIRE].

[3] SNLS collaboration, P. Astier et al., The Supernova legacy survey: Measurement of $\Omega_{M}, \Omega_{\Lambda}$ and $w$ from the first year data set, Astron. Astrophys. 447 (2006) 31 [astro-ph/0510447] [INSPIRE].

[4] A.G. Riess et al., New Hubble Space Telescope Discoveries of Type Ia Supernovae at $z \geq 1$ : Narrowing Constraints on the Early Behavior of Dark Energy, Astrophys. J. 659 (2007) 98 [astro-ph/0611572] [INSPIRE].

[5] T.M. Davis et al., Scrutinizing Exotic Cosmological Models Using ESSENCE Supernova Data Combined with Other Cosmological Probes, Astrophys. J. 666 (2007) 716 [astro-ph/0701510] [INSPIRE].

[6] Supernova Cosmology Project collaboration, M. Kowalski et al., Improved Cosmological Constraints from New, Old and Combined Supernova Datasets, Astrophys. J. 686 (2008) 749 [arXiv: 0804.4142] [INSPIRE].

[7] R. Amanullah et al., Spectra and Light Curves of Six Type Ia Supernovae at $0.511<z<1.12$ and the Union2 Compilation, Astrophys. J. 716 (2010) 712 [arXiv:1004.1711] [INSPIRE].

[8] N. Suzuki et al., The Hubble Space Telescope Cluster Supernova Survey: V. Improving the Dark Energy Constraints Above $z>1$ and Building an Early-Type-Hosted Supernova Sample, Astrophys. J. 746 (2012) 85 [arXiv: 1105.3470] [INSPIRE].

[9] WMAP collaboration, E. Komatsu et al., Seven-Year Wilkinson Microwave Anisotropy Probe (WMAP) Observations: Cosmological Interpretation, Astrophys. J. Suppl. 192 (2011) 18 [arXiv: 1001.4538] [INSPIRE].

[10] D. Larson et al., Seven-Year Wilkinson Microwave Anisotropy Probe (WMAP) Observations: Power Spectra and WMAP-Derived Parameters, Astrophys. J. Suppl. 192 (2011) 16 [arXiv:1001.4635] [INSPIRE].

[11] Planck collaboration, P.A.R. Ade et al., Planck 2013 results. XVI. Cosmological parameters, Astron. Astrophys. 571 (2014) A16 [arXiv:1303.5076] [INSPIRE]. 
[12] SDSS collaboration, D.J. Eisenstein et al., Detection of the Baryon Acoustic Peak in the Large-Scale Correlation Function of SDSS Luminous Red Galaxies, Astrophys. J. 633 (2005) 560 [astro-ph/0501171] [INSPIRE].

[13] W.J. Percival et al., Measuring the Baryon Acoustic Oscillation scale using the SDSS and 2dFGRS, Mon. Not. Roy. Astron. Soc. 381 (2007) 1053 [arXiv:0705.3323] [INSPIRE].

[14] D. Schlegel, M. White and D. Eisenstein with input from the SDSS-III collaboration, The Baryon Oscillation Spectroscopic Survey: Precision measurements of the absolute cosmic distance scale, arXiv:0902.4680 [INSPIRE].

[15] SDSS collaboration, D.J. Eisenstein et al., SDSS-III: Massive Spectroscopic Surveys of the Distant Universe, the Milky Way Galaxy and Extra-Solar Planetary Systems, Astron. J. 142 (2011) 72 [arXiv: 1101.1529] [INSPIRE].

[16] BOSS collaboration, K.S. Dawson et al., The Baryon Oscillation Spectroscopic Survey of SDSS-III, Astron. J. 145 (2013) 10 [arXiv:1208.0022] [INSPIRE].

[17] O. Farooq, D. Mania and B. Ratra, Hubble parameter measurement constraints on dark energy, Astrophys. J. 764 (2013) 138 [arXiv:1211.4253] [INSPIRE].

[18] O. Farooq and B. Ratra, Hubble parameter measurement constraints on the cosmological deceleration-acceleration transition redshift, Astrophys. J. 766 (2013) L7 [arXiv:1301. 5243] [INSPIRE].

[19] O. Farooq, F.R. Madiyar, S. Crandall and B. Ratra, Hubble Parameter Measurement Constraints on the Redshift of the Deceleration-acceleration Transition, Dynamical Dark Energy and Space Curvature, Astrophys. J. 835 (2017) 26 [arXiv:1607.03537] [INSPIRE].

[20] S. Weinberg, The Cosmological Constant Problem, Rev. Mod. Phys. 61 (1989) 1 [INSPIRE].

[21] T. Padmanabhan, Cosmological constant: The Weight of the vacuum, Phys. Rept. 380 (2003) 235 [hep-th/0212290] [INSPIRE].

[22] M. Davis, G. Efstathiou, C.S. Frenk and S.D.M. White, The Evolution of Large Scale Structure in a Universe Dominated by Cold Dark Matter, Astrophys. J. 292 (1985) 371 [INSPIRE].

[23] G. Bertone, Particle Dark Matter: Observations, Models and Searches, Cambridge University Press, Cambridge U.K. (2010).

[24] D.H. Weinberg, M.J. Mortonson, D.J. Eisenstein, C. Hirata, A.G. Riess and E. Rozo, Observational Probes of Cosmic Acceleration, Phys. Rept. 530 (2013) 87 [arXiv:1201.2434] [INSPIRE].

[25] G.S. Sharov and E.G. Vorontsova, Parameters of cosmological models and recent astronomical observations, JCAP 10 (2014) 057 [arXiv: 1407.5405] [INSPIRE].

[26] P.J.E. Peebles and B. Ratra, The Cosmological constant and dark energy, Rev. Mod. Phys. 75 (2003) 559 [astro-ph/0207347] [INSPIRE].

[27] V. Sahni and A. Starobinsky, Reconstructing Dark Energy, Int. J. Mod. Phys. D 15 (2006) 2105 [astro-ph/0610026] [INSPIRE].

[28] M.S. Volkov, Exact self-accelerating cosmologies in the ghost-free bigravity and massive gravity, Phys. Rev. D 86 (2012) 061502 [arXiv:1205.5713] [INSPIRE].

[29] M. Milgrom, A Modification of the Newtonian dynamics as a possible alternative to the hidden mass hypothesis, Astrophys. J. 270 (1983) 365 [INSPIRE].

[30] M. Milgrom, A Modification of the Newtonian dynamics: Implications for galaxies, Astrophys. $J .270$ (1983) 371 [INSPIRE].

[31] G. Gentile, B. Famaey and W.J.G. de Blok, THINGS about MOND, Astron. Astrophys. 527 (2011) A76 [arXiv: 1011.4148] [INSPIRE]. 
[32] T.P. Sotiriou and V. Faraoni, $f(R)$ Theories Of Gravity, Rev. Mod. Phys. 82 (2010) 451 [arXiv:0805.1726] [INSPIRE].

[33] J.-Q. Guo and A.V. Frolov, Cosmological dynamics in $f(R)$ gravity, Phys. Rev. D 88 (2013) 124036 [arXiv:1305.7290] [INSPIRE].

[34] S. Capozziello and M. De Laurentis, Extended Theories of Gravity, Phys. Rept. 509 (2011) 167 [arXiv:1108.6266] [INSPIRE].

[35] L. Randall and R. Sundrum, An Alternative to compactification, Phys. Rev. Lett. 83 (1999) 4690 [hep-th/9906064] [INSPIRE].

[36] A. Falkowski, Z. Lalak and S. Pokorski, Supersymmetrizing branes with bulk in five-dimensional supergravity, Phys. Lett. B 491 (2000) 172 [hep-th/0004093] [INSPIRE].

[37] P. Binetruy, C. Deffayet and D. Langlois, Nonconventional cosmology from a brane universe, Nucl. Phys. B 565 (2000) 269 [hep-th/9905012] [InSPIRE].

[38] T. Shiromizu, K.-i. Maeda and M. Sasaki, The Einstein equation on the 3-brane world, Phys. Rev. D 62 (2000) 024012 [gr-qc/9910076] [INSPIRE].

[39] J.M. Cline, C. Grojean and G. Servant, Cosmological expansion in the presence of extra dimensions, Phys. Rev. Lett. 83 (1999) 4245 [hep-ph/9906523] [INSPIRE].

[40] T. Damour and A.M. Polyakov, String theory and gravity, Gen. Rel. Grav. 26 (1994) 1171 [gr-qc/9411069] [INSPIRE].

[41] J.M. Overduin and P.S. Wesson, Kaluza-Klein gravity, Phys. Rept. 283 (1997) 303 [gr-qc/9805018] [INSPIRE].

[42] M. Visser, Jerk and the cosmological equation of state, Class. Quant. Grav. 21 (2004) 2603 [gr-qc/0309109] [INSPIRE].

[43] M. Visser, Cosmography: Cosmology without the Einstein equations, Gen. Rel. Grav. 37 (2005) 1541 [gr-qc/0411131] [INSPIRE].

[44] C. Shapiro and M.S. Turner, What do we really know about cosmic acceleration?, Astrophys. J. 649 (2006) 563 [astro-ph/0512586] [INSPIRE].

[45] R.D. Blandford, M.A. Amin, E.A. Baltz, K. Mandel and P.J. Marshall, Cosmokinetics, ASP Conf. Ser. 339 (2005) 27 [astro-ph/0408279] [INSPIRE].

[46] Ø. Elgarøy and T. Multamäki, Bayesian analysis of Friedmannless cosmologies, JCAP 09 (2006) 002 [astro-ph/0603053] [INSPIRE].

[47] D. Rapetti, S.W. Allen, M.A. Amin and R.D. Blandford, A kinematical approach to dark energy studies, Mon. Not. Roy. Astron. Soc. 375 (2007) 1510 [astro-ph/0605683] [InSPIRE].

[48] J.V. Cunha and J.A.S. Lima, Transition Redshift: New Kinematic Constraints from Supernovae, Mon. Not. Roy. Astron. Soc. 390 (2008) 210 [arXiv:0805.1261] [InSPIRE].

[49] A.C.C. Guimaraes, J.V. Cunha and J.A.S. Lima, Bayesian Analysis and Constraints on Kinematic Models from Union SNIa, JCAP 10 (2009) 010 [arXiv:0904.3550] [INSPIRE].

[50] N. Rani, D. Jain, S. Mahajan, A. Mukherjee and N. Pires, Transition Redshift: New constraints from parametric and nonparametric methods, JCAP 12 (2015) 045 [arXiv: 1503.08543] [INSPIRE].

[51] L. Xu, W. Li and J. Lu, Constraints on Kinematic Model from Recent Cosmic Observations: SN Ia, BAO and Observational Hubble Data, JCAP 07 (2009) 031 [arXiv:0905.4552] [INSPIRE].

[52] R.F.L. Holanda, J.S. Alcaniz and J.C. Carvalho, Cosmography with the Sunyaev-Zeldovich effect and X-ray data, JCAP 06 (2013) 033 [arXiv: 1303.3307] [INSPIRE].

[53] A. Sandage, Cosmology: A search for two numbers, Phys. Today 23 (1970) 34. 
[54] J.A.S. Lima, J.F. Jesus, R.C. Santos and M.S.S. Gill, Is the transition redshift a new cosmological number?, arXiv: 1205.4688 [INSPIRE].

[55] M. Moresco et al., A 6\% measurement of the Hubble parameter at $z \sim 0.45$ : direct evidence of the epoch of cosmic re-acceleration, JCAP 05 (2016) 014 [arXiv:1601.01701] [INSPIRE].

[56] SDSS collaboration, M. Betoule et al., Improved cosmological constraints from a joint analysis of the SDSS-II and SNLS supernova samples, Astron. Astrophys. 568 (2014) A22 [arXiv: 1401.4064] [INSPIRE].

[57] M. Abramowitz and I.A. Stegun, Handbook of mathematical functions: with formulas, graphs, and mathematical tables. Volume 55, Applied Mathematics Series, Courier Corporation (1964).

[58] G.S. Sharov and E.G. Vorontsova, Parameters of cosmological models and recent astronomical observations, JCAP 10 (2014) 057 [arXiv:1407.5405] [INSPIRE].

[59] J.F. Jesus, T.M. Gregório, F. Andrade-Oliveira, R. Valentim and C.A.O. Matos, Bayesian correction of $H(z)$ data uncertainties, Mon. Not. Roy. Astron. Soc. 477 (2018) 2867 [arXiv: 1709.00646] [INSPIRE].

[60] SDSS collaboration, M. Sako et al., The Data Release of the Sloan Digital Sky Survey-II Supernova Survey, arXiv:1401.3317 [INSPIRE].

[61] SNLS collaboration, A. Conley et al., Supernova Constraints and Systematic Uncertainties from the First 3 Years of the Supernova Legacy Survey, Astrophys. J. Suppl. 192 (2011) 1 [arXiv: 1104.1443] [INSPIRE].

[62] SNLS collaboration, J. Guy et al., SALT2: Using distant supernovae to improve the use of Type Ia supernovae as distance indicators, Astron. Astrophys. 466 (2007) 11 [astro-ph/0701828] [INSPIRE].

[63] SNLS collaboration, J. Guy et al., The Supernova Legacy Survey 3-year sample: Type Ia Supernovae photometric distances and cosmological constraints, Astron. Astrophys. 523 (2010) A7 [arXiv: 1010.4743] [INSPIRE].

[64] SDSS collaboration, M. Betoule et al., Improved cosmological constraints from a joint analysis of the SDSS-II and SNLS supernova samples, Astron. Astrophys. 568 (2014) A22 [arXiv: 1401.4064] [INSPIRE].

[65] J. Goodman and J. Weare, Ensemble samplers with affine invariance, Commun. Appl. Math. Comput. Sci. 5 (2010) 65.

[66] D. Foreman-Mackey, D.W. Hogg, D. Lang and J. Goodman, emcee: The MCMC Hammer, Publ. Astron. Soc. Pac. 125 (2013) 306 [arXiv:1202.3665] [InSPIRE].

[67] R. Allison and J. Dunkley, Comparison of sampling techniques for Bayesian parameter estimation, Mon. Not. Roy. Astron. Soc. 437 (2014) 3918 [arXiv:1308.2675] [INSPIRE].

[68] A. Lewis and S. Bridle, Cosmological parameters from CMB and other data: A Monte Carlo approach, Phys. Rev. D 66 (2002) 103511 [astro-ph/0205436] [INSPIRE].

[69] A.G. Riess et al., A 3\% Solution: Determination of the Hubble Constant with the Hubble Space Telescope and Wide Field Camera 3, Astrophys. J. 730 (2011) 119 [Erratum ibid. 732 (2011) 129] [arXiv:1103.2976] [INSPIRE].

[70] A.G. Riess et al., A 2.4\% Determination of the Local Value of the Hubble Constant, Astrophys. J. 826 (2016) 56 [arXiv:1604.01424] [INSPIRE].

[71] Planck collaboration, P.A.R. Ade et al., Planck 2015 results. XIII. Cosmological parameters, Astron. Astrophys. 594 (2016) A13 [arXiv:1502.01589] [InSPIRE].

[72] J.L. Bernal, L. Verde and A.G. Riess, The trouble with $H_{0}$, JCAP 10 (2016) 019 [arXiv: 1607.05617] [INSPIRE]. 